\title{
Therapy Related Acute Lymphoblastic Leukemia (t-ALL): Case Report and Literature Review
}

\author{
Asma Ameen*, Nida Anwar, Tahir Sultan Shamsi \\ Department of Adult Clinical Hematology, National Institute of Blood Disease \& Bone Marrow Transplantation, Karachi, Pakistan.
}

\begin{abstract}
Therapy related Acute Lymphoblastic Leukemia (t-ALL) is a rare secondary malignancy. It occurs in patients who are exposed to chemotherapy and/or radiotherapy for primary malignancy. We are reporting here a case of a thirty five years old female patient who was referred to us for fever, malaise and bleeding from gums. She had a history of ovarian carcinoma for which she took carboplatin and other chemotherapeutic agents. Extensive workup was done which was consistent with the diagnosis of Precursor-B cell Acute Lymphoblastic Leukemia. In the context of previous history of exposure to carboplatin, she was diagnosed as a case of therapy related Acute Lymphoblastic Leukemia secondary to carboplatin.
\end{abstract}

Keywords: Carboplatin, Therapy related ALL, Ovarian cancer, Secondary malignancy, Platinum based chemotherapy, Radiotherapy.

\section{INTRODUCTION}

Therapy related Acute Lymphoblastic Leukemia (t-ALL) is a rare clinical entity. It occurs following chemotherapy and/or radiotherapy for primary malignancies $[1,2]$.

Therapy related acute lymphoblastic leukemia (t-ALL) which represents approximately $2-10 \%$ of all cases of diagnosed ALL is seen much less frequently than therapy related AML [3]. It most commonly occurs after therapy with alkylating agents or DNA topoisomerase II inhibitors [4]. It usually occurs at later age than de-novo ALL (median age 60 years) [5]. Platinum based chemotherapy agents which are the cornerstone of treatment of solid organ tumors such as ovarian, testicular, bladder, lung and head $\&$ neck have been found to be carcinogenic and mutagenic [6]. The incidence of such secondary malignancies are on a rising trend and is associated with a grave prognosis $[7,8]$. We are reporting here a case of chemotherapy induced Acute Lymphoblastic Leukemia in a patient who was diagnosed with ovarian carcinoma and received carboplatin in induction as well as maintenance chemotherapy regimen.

\section{CASE REPORT}

A 35 years old female patient was referred with the concern of intermittent fever, malaise and bleeding gums. She was diagnosed as a case of ovarian carcinoma in November 2013 post delivery of her second child. She then received 6 cycles of carboplatin and paclitaxel every 21 days followed by a maintenance therapy of 3 cycles of carboplatin. Remission was achieved in the following 6 months. She was then monitored for her disease by a surveillance $\mathrm{CT}$ scan done every 3 months. In August 2016, her surveillance CT scan showed multiple deposits in liver, colon and rectum which were suggestive of recurrence of the primary disease with

*Address correspondence to this author at the Department of Adult Clinical Hematology, National Institute of Blood Disease \& Bone Marrow Transplantation, Karachi, Pakistan. E-mail: asma.ameen.docrat@gmail.com metastasis. She was again prescribed with carboplatin and paclitaxel along with bevacizumab (3 cycles). She achieved second remission in December 2016 and was kept on maintenance with pazopanib. She took pazopanib for some time but showed noncompliance and left the treatment. Her scan of December 2017, showed recurrence of the disease. She was again started with the same three drugs i.e: carboplatin, paclitaxel and bevacizumab given previously and her surveillance scan showed stable disease. In May 2018, she developed fever and bleeding from gums. Her CBC demonstrated high WBC count for which she was started on hydroxyurea and allopurinol and referred to us. Peripheral smear revealed presence of blast cells and subsequent peripheral blood imunophenotyping revealed CD $19(+) \mathrm{CD}$ $45(+) \mathrm{CD} 79 \mathrm{a}(+) \mathrm{Tdt}(+)$; features favouring Precursor B-cell Acute Lymphoblastic Leukemia according to WHO criteria.

Her Bone marrow cytogenetics turned out to be normal female karyotype (46 XX). Prognostic markers such as BCR-ABL p190 by PCR, MLL gene by FISH and Del 17p53 by FISH were all negative. No mutation was detected on NGS panel for inherited disease. After establishing the diagnosis of Precursor B-cell ALL, we started her with poor risk UK-ALL $\mathrm{XI}$ induction protocol comprising of 28 days. She was doing well initially but during the course of her treatment she developed invasive fungal chest infection and subacute intestinal obstruction. Patient was given antiviral prophylaxis, regular mouthcare and broad spectrum antibiotics. She was also given amphotericin for her invasive fungal infection. Subacute intestinal obstruction was dealt with giving rest to the gut by keeping her nil per oral. She has been discharged after the completion of induction protocol and after alleviation of her symptoms.

\section{DISCUSSION}

Therapy related Acute Lymphoblastic Leukemia is a type of secondary hematological malignancy which is an emerging 
long term complication of prior therapy to cytotoxic agents (chemotherapy and radiotherapy). Its incidence is on a rising trend. Therapy related myeloid neoplasms (MN's) is a well-defined category in WHO classification of myeloid neoplasms but therapy related Acute Lymphoblastic Leukemia is a rare entity and till this date it has not been fully recognized due to limited available case reports $[1,9]$.

Platinum based chemotherapeutic agents are commonly used for the treatment of solid organ tumors such as ovary, breast, bladder, lung, head \& neck and endometrium. They play a pivotal role in the treatment of ovarian cancer. Carboplatin and Cisplastin are among the most important drugs used for the treatment of ovarian cancer since the past three decades. Our Literature search shows that platinum based chemotherapeutic agents have carcinogenic potential in-vitro. It causes intrastrand and interstrand DNA cross-links similar to conventional alkylating agents leading to DNA instability and leukomegenic potential. The risk depends on the cumulative dose of the drug and duration of the treatment [6].

In our case patient had an exposure to carboplatin for a number of years as a part of her treatment for her primary malignancy (ovarian cancer). Our literature review reveals that platinum based chemotherapeutic agents confer risk of secondary ALL in a subset of patients. In a case control study done in North America and Europe, it was observed that risk of leukemia was significantly increased in women who were exposed to platinum based chemotherapeutic agents for ovarian cancer as compared to other agents. In this case control study 94 percent patients of secondary leukemia were treated with alkylating drugs or platinum based drugs as compare to 65 percent of control patients. The patients with leukemia received a median of nine cycles of cisplatin or seven cycles of carboplatin, women in the control group received a median of seven cycles of cisplatin or six cycles of carboplatin. The risk of leukemia was significantly increased after treatment with platinum (relative risk, 4.0; 95 percent confidence interval, 1.4 to 11.4 ) but was considerably lower than the risk after treatment with other drugs (relative risk, 20.8; 95 percent confidence interval, 6.3 to 68.3 ).

The risk of leukemia increased with the duration of platinum-based chemotherapy, with a relative risk of 7.0 among women who were treated for more than 12 months ( $\mathrm{P}$ for trend $=0.001$ ) [5]. Our patient received 13 cycles of carboplatin throughout her course of disease which increased her risk of conferring leukemia in context of the case report mentioned.

\section{CONCLUSION}

Till this date, no specific criterion has been published for the diagnosis of therapy related Acute Lymphoblastic Leukemia. Because of the lack of availability of such criteria, we are not able to study or classify this entity of leukemia. There is an acute need for the diagnostic and prognostic classification of this disease entity for institution of optimal patient and disease tailored treatment.

\section{CONFLICT OF INTEREST}

Declared none.

\section{ACKNOWLEDGEMENTS}

Declared none.

\section{REFERENCES}

[1] Aldoss I, Stiller T, Tsai NC, et al. Therapy-related acute lymphoblastic leukemia has distinct clinical and cytogenetic features compared to de novo acute lymphoblastic leukemia, but outcomes are comparable in transplanted patients. Haematologica 2018; 103(10): 1662-8.

DOI: $10.3324 /$ haematol.2018.193599

[2] Kelleher N, Gallardo D, González-Campos J, et al. Incidence, clinical and biological characteristics and outcome of secondary acute lymphoblastic leukemia after solid organ or hematologic malignancy. Leuk Lymphoma 2016; 57(1): 86-91.

DOI: $10.3109 / 10428194.2015 .1040013$

[3] Swaika A, Frank RD, Yang D, et al. Second primary acute lymphoblastic leukemia in adults: A SEER analysis of incidence and outcomes. Cancer Med 2018; 7(2): 499-507. DOI: $10.1002 /$ cam4.1266

[4] Abdul wahab A, Sykes J, Kamel-Reid S, Chang H, Brandwein JM. Therapy-related acute lymphoblastic leukemia is more frequent than previously recognized and has a poor prognosis. Cancer 2012; 118(16): 3962-7. DOI: 10.1002/cncr.26735

[5] Travis LB, Holowaty EJ, Bergfeldt K, et al. Risk of leukemia after platinum-based chemotherapy for ovarian cancer. New Engl J Med 1999; 340(5): 351-7.

DOI: 10.1056/NEJM199902043400504

[6] Chen W, Wang E, Lu Y, Gaal KK, Huang Q. Therapy-related acute lymphoblastic leukemia without 11q23 abnormality: Report of six cases and a literature review. Am J Clin Pathol 2010; 133(1): 75-82. DOI: 10.1309/AJCPYWC6AQC7BAVJ

[7] Giri S, Chi M, Johnson B, et al. Secondary acute lymphoblastic leukemia is an independent predictor of poor prognosis. Leuk Res 2015; 39(12): 1342-6.

DOI: 10.1016/j.leukres.2015.09.011

[8] Gallardo D, Brunet S, Montesinos P, Ribera JM, Barba P, Garcia O. Therapy related acute lymphoblastic leukemia: Pethema experience. Blood 2013; 122: 4994.

[9] Ferraro F, Gao F, Stockerl-Goldstein K, Westervelt P, DiPersio JF, Ghobadi A. Secondary acute lymphoblastic leukemia, a retrospective analysis from Washington University and meta-analysis of published data. Leuk Res 2018; 72: 86-91. DOI: 10.1016/j.leukres.2018.07.024 